\title{
Neuronavigation system in neurosurgery: Technical details and our early clinical experiences
}

\author{
Rajiv Jha $M C h^{1}$, Bikesh Khambu $M C h^{2}$, Rajendra Shrestha $P h D^{3}$, Prakash Bista $M S^{4}$ \\ ${ }^{1,2,3,4}$ National Neurosurgical Referral Center, NAMS Bir Hospital, Kathmandu, Nepal
}

\section{Abstract}

Introduction: Image-guided surgery is the need of time in neurosurgery. The use of neuronavigation has a significant impact on various neurosurgical procedures. We report our clinical experience and surgical techniques of neuronavigation assisted intracranial and spinal surgeries.

Materials and Method: This is observational longitudinal study of 65patients who underwent surgeries using navigation system in the department of neurosurgery, National Neurosurgical Referral Center, National Academy of Medical Sciences (NAMS) Bir hospital and National Trauma Center, over the period of one and a half year. Description of Navigation techniques, its applications and surgical outcome were studied. Medtronic Stealth station 7 and Brain Lab Curve Neuronavigation system, which can be used as both frame based and frameless image guided system were employed in this study.

Results: We used Neuronavigation system in 60 cases of cranial procedures and 5 cases of spinal stabilizing procedures. Among intracranial surgeries we utilized this system to effectively make bone flaps and burr holes, to detect critically located deep-seated, subcortical and skull base tumors and to operate on intra parenchymal lesions with grossly unclear margins.

Neuronavigation system was used in 5 cases of spinal procedures for pedicle screw placement.

Conclusion: Neuronavigation is a tool that provides numerous advantages to the neurosurgeon like more accurate planning approach, ability to use smaller approaches, higher precision for intraoperative localization of different anatomical structures and offering greater surgical safety. This technique, although apparently very complex in the beginning, becomes very user-friendly gradually and positively appreciated by everyone who uses it, after going through a relatively short learning curve.

Key words: Brain navigation, Neuronavigation, Intra operative imaging, Spinal navigation

Access this article online
Website: https://www.nepjol.info/index.php/NJN
DOI: https://doi.org/10.3126/njn.v18i1.35017
HOW TO CITE
Jha R, Khambu B, Shrestha R, Bista P. Neuronavigation system in
neurosurgery: Technical details and our early clinical experiences.
NJNS. 2021;18(1):45-50.

'ORCID id: 0000-0002-2830-7835

${ }^{2}$ ORCID id: 0000-0002-7369-589X

${ }^{3}$ ORCID id: 0000-0003-2097-0699

${ }^{4}$ ORCID id: 0000-0002-4811-6964

Address for correspondence:

Prof. Dr. Rajiv Jha

National Neurosurgical Referral Center, NAMS Bir Hospital

Kathmandu, Nepal

Tel: +977-9851039699

E-mail:medrajiv18@hotmail.com

Copyright (C) 2021 Nepalese Society of Neurosurgeons (NESON)

ISSN: 1813-1948 (Print), 1813-1956 (Online)

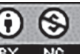

This work is licensed under a Creative Commons Attribution-Non Commercial 4.0 International License.

\section{Introduction}

Teurosurgery will never be the same after the 1 introduction of Neuronavigation. The possibilities for the application of Neuronavigation to contemporary neurosurgery are practically unlimited, with the potential to supply better guidance, orientation, and localization, and hence a higher confidence level for the surgeon and an improved outcome for the patient. ${ }^{1,2,3,4}$

Neuronavigation is the set of computer-assisted technologies used by neurosurgeons to guide or navigate within the borders of the skull or vertebral column during surgery. As a term Neuronavigation is synonymous with image-guided surgery, computer-assisted or computeraided surgery. This technique dates back to the early 1900 s and that gained popularity during the 1940s, especially with the development of surgery for the treatment of movement disorders such as Parkinson's disease and dystonia those needs to be reached certain exact locations. Over the past decade, brain surgeons have been accustomed to use intraoperative surgical navigation 
as a technological means to improve surgical outcomes and to reduce surgical morbidity. ${ }^{5}$

Neuronavigation provides intraoperative orientation to the surgeons and helps them in planning a precise surgical approach to the targeted lesion and defines the surrounding neurovascular structures. By incorporation of the functional data provided by functional MRI and magnetoencephalography with Neuronavigation, it also guides to avoid the eloquent areas of the brain during surgery. ${ }^{6}$ Neuronavigation provides the surgeon the ability to see a patient's anatomy in three dimensions and accurately pinpoint a location in the brain or spinal cord with the aid of diagnostic images such as computed tomography (CT) and magnetic resonance imaging (MRI), or intraoperative images like ultrasound, MRI, etc. It also enables surgeons to track instruments in relation to a patient's anatomy and track the anatomy itself during a surgical procedure.

Neuronavigation utilizes the principle of stereotaxis. The brain is considered as a geometric volume which can be divided by three imaginary intersecting spatial planes, orthogonal to each other (horizontal, frontal and sagittal) based on the Cartesian coordinate system. Any point within the brain can be specified by measuring its distance along these three intersecting planes. Neuronavigation provides a precise surgical guidance by referencing this coordinate system of the brain with a parallel coordinate system of the three-dimensional image data of the patient that is displayed on the console of the computer-workstation so that the medical images become point-to-point maps of the corresponding actual locations within the brain .?

\section{Materials and Method}

This is an observational longitudinal study and technical details of neuronavigation guided different surgical procedure in the brain and spine. This study was taken place at National Neurosurgical Referral Center, Department of Neurosurgery Bir hospital and National Trauma Center (NTC), National Academy of Medical Sciences (NAMS) between July 2018 till December 2019. Institutional review board (IRB) approval was taken from the hospitals for the study. Consent was taken from the patients if they were able to write and from the next of kin if they were not able to give consent. There were no such indications or contraindications of its use, we are trying to use it in maximum number of cases so that precise bone work, dural opening and tumour localization and excision can be achieved. Only the cases which undergone use of intraoperative navigation system were included in this study. Details of all navigation procedures, types of surgical procedures and its final outcome were analyzed.
Placement of pedicle screw was assessed with an intraoperative CT scan using Laine's grading system.

\section{Procedure}

For cranial surgery, prior to the procedure the patient gets an MRI scan and/or CT scan with fiducials pasted on the scalp. These images are stored on an optical disc/ external drive. This disc/external drive is then transferred to the navigation machine to be used in the operating theatre (Figure 1a). The fiducials have to stay on the patient. Thus, the imaging is done either the evening before or the day of the cranial surgery. (Figure 1b) Once in the operating room, the external markers are registered for accuracy. The patient is then draped and prepped for surgery. Standard skull landmarks are then correlated for accuracy using a probe that has been registered on the navigation system. The provided probe or other instruments (depending on the system used and its features) can then be registered for intraoperative navigation to the target. (Figure 1c) Prior to the start of the procedure, the craniotomy can be planned to be right over the tumor and thus minimize the size of the bone flap (Figure 1d). Intraoperatively, the approach to the tumor can be seen in order to avoid vital brain structures and minimize morbidity to the brain. In addition, the extent of the tumor resection can be monitored indirectly by correlating the position of the instruments with the preoperative imaging studies (Figure 1e).

During the spinal procedures, the fiducials for registration were put on spinous process of the spine. A clamp is placed on the spinous process near the operative area in the spine at least 2 level above or below (Figure 2a). This clamp has to remain stable throughout the procedure otherwise the accuracy of the navigation can be diminished. Further Brain Lab intraoperative CT scan was positioned to obtain three-dimensional (3D) images, (Figure 2b) which were transferred to the navigation system for automatic registration, and the image-guided probe was used to confirm the entry point and orientation of pedicle screws. The pedicles were cannulated, and the trajectories were visualized on sagittal, axial, and coronal images at the navigation displayed images (Figure 2c). The appropriate screw was selected and inserted.

\section{Results}

Total number of procedures with intraoperative use of navigation were 65 , of which majority were cranial procedures (92\%). In about $90 \%$ of the cases, Navigation was used for tumor excision and biopsy (Table 1).

We have used navigation in all the cases of trans sphenoidal endoscopic pituitary surgeries (Table 2). 
Neuronavigation system in neurosurgery

Neuronavigation was used to take the biopsy in 22 cases, of which biopsy was conclusive in $91 \%$.

Glial tumors, meningiomas and pineal region tumors could be excised $100 \%$ while in pituitary tumors, complete excision achieved in only $92 \%$.

In all the cases, craniotomy size, opening of the dura and tumor localization with its surrounding margins were very accurate. None of these procedures required revision in doing bone and dura work.

In the cases where we thought surgery takes very precise positioning with longer duration or in posterior fossa tumors, we put patient in 3 pins otherwise all cases we have done without head fixation. Use of neuronavigation process needs extra time about 30-40 minutes, which was initially more time consuming but gradually with increasing use, the time utilized for navigation

\begin{tabular}{l|c|c|}
\hline & $\begin{array}{c}\text { Number of } \\
\text { cases }\end{array}$ & $\%$ \\
\hline Tumor Excision & 36 & $55 \%$ \\
\hline Tumor Biopsy & 22 & $34 \%$ \\
\hline Catheter placement & 1 & $1.5 \%$ \\
\hline Cyst Aspiration & 1 & $1.5 \%$ \\
\hline Pedicle Screw Placement & 5 & $8 \%$ \\
\hline
\end{tabular}

Table 1. Neuronavigation guided procedures

\begin{tabular}{l|c|c}
\hline Pituitary Tumors & 24 & $40 \%$ \\
Glial Tumors & 6 & $10 \%$ \\
\hline Meningiomas & 5 & $8 \%$ \\
\hline Pineal region Tumors & 1 & $2 \%$ \\
\hline
\end{tabular}

Table 2. Navigation used in different brain tumor surgeries became less.
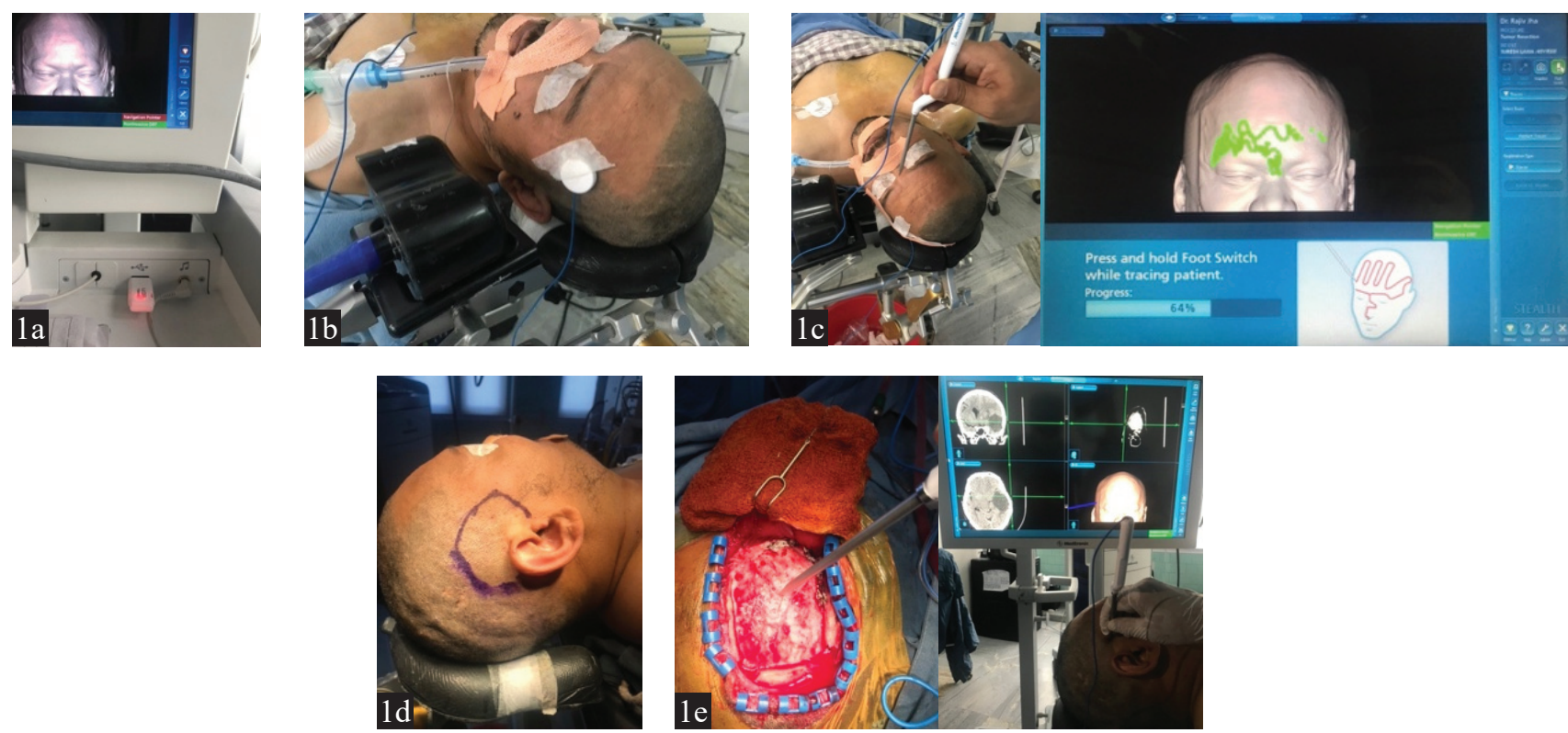

Figure 1(a) Pre-operative CT/MRI images are transferred to navigation machine. Figure 1(b) Fiducial over the scalp, Figure 1(c) Registration at different craniometric points. Figure 1(d). Precise selection of craniotomy size. Figure 1(e). Intraoperative visualization of extent of tumor
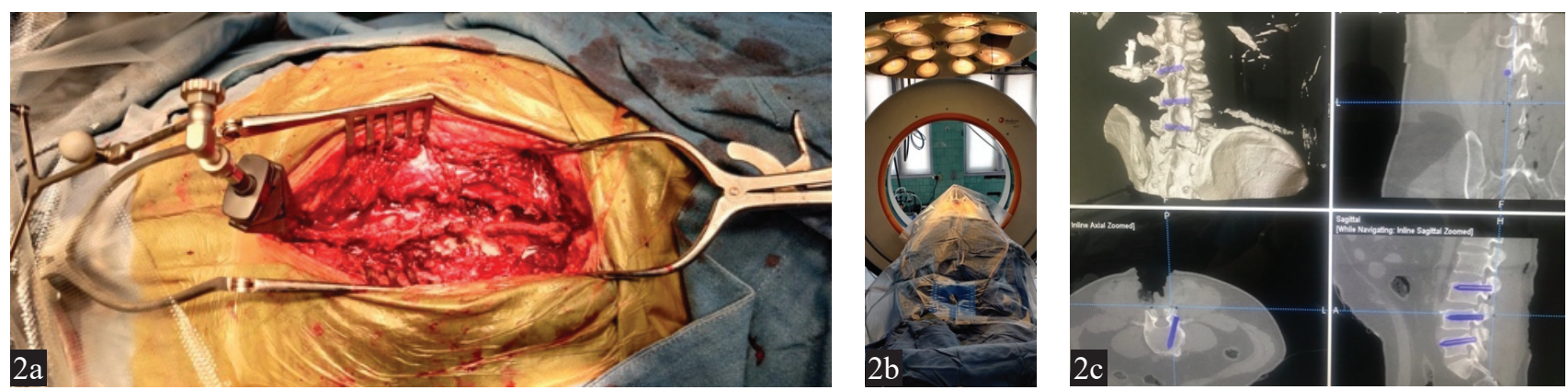

Figure 2(a) Metallic Fudicial over the spinous process. Figure 2(b) Obtaining 3D images using intra operative CT scan. Figure 2(c) Pedicle screw placement using navigation. 
Unsterile atmosphere

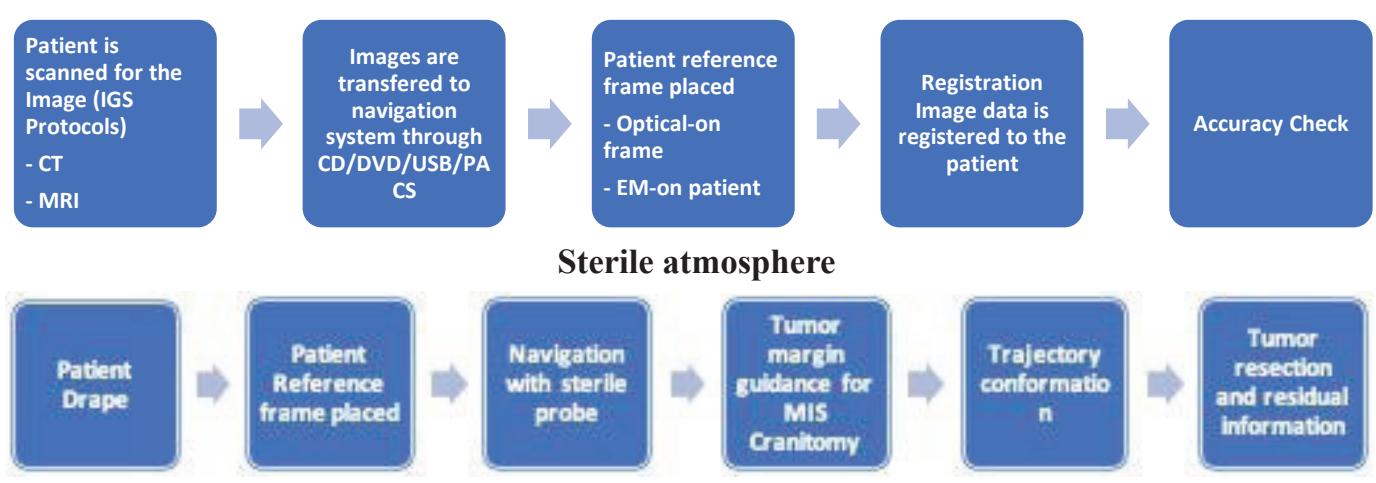

\section{Unsterile Atmosphere}

1. Patient is scanned for the Image under IGS recommended Protocol for various modalities of Medical Imaging

IGS Protocol for Patient Imaging

\section{Before Scan}

Separate the patient's head from the scanner headrest with towels or foam. Contrast agents may be injected before scanning.

\section{CT Scans Only}

- Standard soft tissue algorithm

- No gantry tilt

- can contiguous slices

- Use $1 \mathrm{~mm}$ slice thickness \& $1 \mathrm{~mm}$ slice interval

- Axial slices are preferred.

- $\quad$ FOV equal to or up to 250

- Use a square image matrix of 256 × 256 or 512 × 512.

\section{MR Scans Only}

- No oblique slices

- Single-echo, no multi-echo scans

- Axial slices are preferred

- Use $1 \mathrm{~mm}$ slice thickness \& $1 \mathrm{~mm}$ Slice interval.

- $\quad \mathrm{T} 1$ or T2 any sequence can be used

- $\quad$ FOV equal to or up to 250

2. Image is transferred to Navigation system through $\mathrm{CD} / \mathrm{DVD} / \mathrm{USB} /$ PACS

Archive the raw scan exam from the scanner to a CD.

Do not archive or transfer scout images

Transfer the scan in an uncompressed file format (exception: compressed scans from GE scanners are acceptable).

3. Patient Reference Frame is placed

To reference frame is placed so that it is fixed with reference to the Patient head. The frame is fixed on the Mayfield clamp for optical system. For EM navigation, reference is attached to the patient head on the opposite of the surgical site.

\section{Registration}

Patient data is registered to the patient.

\section{Sterile Atmosphere}

1. Patient Drape

Patient is draped

2. Sterile Reference Frame placed Sterile Reference Frame is switched to unsterile Reference Frame

3. Navigation with sterile probe Navigation is done using a sterile probe

4. Tumor Margin guidance for MIS Craniotomy

3D model of tumor is created using Seed, Threshold and Manual.

5. Trajectory conformation Trajectory for the procedure is confirmed by confirming target and the entry. The trajectory should be such that there are no critical structures on the way.

6. Tumor resection and residual information

Tumor is resected and checked if all the tumor is resected as planned. 
Registration Technique

a. Touch

It is Fiducial based. For this method 4-12 Fiducial markers are stick to the patient head before scan, so that the scan it is included in the patient image. During registration patient is registered by touching on the center of these Fiducial markers. Minimum 4 is required but it is recommended place more up to 12 , as it increase the accuracy.

b. Trace

It is Fiducial less technique, done by surface countering. Probe trace on the patient head without removing the probe from patient skin starting from tip of nose, left ridge line and then to right and then paint on to the patient skull until registration complete.

5. Accuracy Check

Check accuracy buy touching the known landmarks on the patient with the probe

Figure 3: IGS Workflow for Cranial Tumors

\section{Discussion}

Frame-based stereotactic target calculation is an established method for localizing intracranial le- sions during an operation. Using this technique, very small tumors visible in CT or MRI can be transferred into the stereotactic space and localized precisely, permitting introduction of a catheter into the lesion. ${ }^{8,9}$ The mechanical and especially the registration accuracy of frame-based systems cannot be equaled by any navigation system. ${ }^{10,11}$ The arrangement of the rods of the localization frame is optimal for excluding errors arising from amplification of the input data, while the calculation of the centers of the rods is possible to one- tenth of a pixel and is independent of slice thickness. ${ }^{12}$ However, the frame-based technique requires more precautions with regard to the fixation of the head ring in order to permit sufficient intraoperative access for suboccipital or subtemporal approaches. Frame-based introduction of a catheter is difficult in all fronto-temporooccipito-basal lesions that, from the microsurgical point of view, are best approached along the skull base to begin with and only approached trans cortically in the vicinity of the lesion. In general, frame-based localization allows only a lateral approach to these lesions with longer transcortical access.

In contrast, mechanical flexibility imposes no restrictions on the microsurgical technique. ${ }^{13,14}$ It can be used interactively at any time during the operation and is not limited by preoperative calculation of only a few target coordinates. Navigation is very easily adapted to endoscopy ${ }^{15,16}$ and can also be used for stereotactic biopsies. ${ }^{17}$ If the introduction of a catheter seems the safest way to reach a tumor, this can be guided by a navigation device as well.

\section{IGS Workflow for Cranial Tumors}

Among the various steps of IGS Workflow few of the steps are perform on unsterile atmosphere and rest on sterile atmosphere (Figure 3).

\section{Conclusion}

Neuronavigation is very helpful tool in neurosurgery. It can be used in literary all elective and emergency surgeries. It provides great accuracy, starting from planning a scalp incision to removing tumors inside the brain. Brain shift is a challenge in few conditions but by using intra-operative imaging systems, this can be eliminated. It not only helps keep patient safe but also gives great confidence to the operating surgeons.

\section{Acknowledgement}

The authors would like to thank the patients and their relatives for providing consent to publish this article. The authors would also like to thank Dr Roshan Khatiwda, Dr Sushil Mohan Bhattarai and Dr Ali Aefee, final year MCh Residents at Department of Neurosurgery, NAMS Bir Hospital for technical help during performing the procedures.

Conflict of Interest: None

Source(s) of support: None 


\section{References}

1. Enchev Y, Bozinov O, Miller D, et al. Imageguided ultrasonography for recurrent cystic gliomas. ActaNeurochir (Wien). 2006;148(10):1053-1063. https://doi.org/10.1007/s00701-006-0858-6

2. Enchev Y, Oi S. Historical trends of neuroendoscopic surgical techniques in the treatment of hydrocephalus. Neurosurg Rev. 2008;31(3):249-262. https://doi.org/10.1007/s10143-008-0131-y

3. Enchev YP, Popov RV, Romansky KV, Marinov MB, Bussarsky VA. Cranial neuronavigation--a step forward or a step aside in modern neurosurgery. Folia Med (Plovdiv). 2008;50(2):5-10.

4. Mandel M, Amorim R, Paiva W, Prudente M, Teixeira MJ, Andrade AF. 3D preoperative planning in the ER with OsiriX ${ }^{\circledR}$ : when there is no time for neuronavigation. Sensors (Basel). 2013;13(5):6477-6491. https://doi.org/10.3390/ s130506477

5. Ganslandt O, Behari S, Gralla J, Fahlbusch R, Nimsky C. Neuronavigation: concept, techniques and applications. Neurol India. 2002;50(3):244-255.

6. Nimsky C, Ganslandt $\mathrm{O}$, Kober $\mathrm{H}$, et al. Integration of functional magnetic resonance imaging supported by magnetoencephalography in functional neuronavigation. Neurosurgery. 1999;44(6):1249-1256. https://doi. org/10.1097/00006123-199906000-00044

7. Ganslandt O, Fahlbusch R, Nimsky C, et al. Functional neuronavigation with magnetoencephalography: outcome in 50 patients with lesions around the motor cortex. Neurosurg Focus. 1999;6(3):e3. https://doi. org/10.3171/foc.1999.6.3.6

8. Boecher-Schwarz HG, Grunert P, Guenthner M, Kessel G, Mueller-Forell W. Stereotactically guided cavernous malformation surgery. Minim
Invasive Neurosurg. 1996;39(2):50-55. https://doi. org/10.1055/s-2008-1052216

9. Ebeling U, Hasdemir MG. Stereotactic guided microsurgery of cerebral lesions. Minim Invasive Neurosurg. 1995;38(1):10-15. https://doi. org/10.1055/s-2008-1053454

10. Darabi K, Grunert P, Perneczky A (1997) Accuracy of intraoperative navigation using skin markers. In Lemke HU, Vannier M W , and Inamura K (eds): CAR ‘97. New York: Elsevier, pp 920-924.

11. Grunert P, Müller-forell W, Darabi K, Reisch R, Busert C, Hopf N, Perneczky A. Basic Principles and Clinical Applications of Neuronavigation and Intraoperative Computed Tomography, Computer Aided Surgery, 1998; 3(4)166-173. https://doi. org/10.3109/10929089809148141

12. Alexander E III,Moriarty TM, Kikinis R, Black $\mathrm{P}$, Jolesz FM. The present and future role of intraoperative MRI in neurosurgical procedures. StereotactFunctNeurosurg1997;68:10-17. https:// doi.org/10.1159/000099896

13. Grunert P,Müller-forell V, Darabi K,Reisch R, Busert C, Hopf N,Perneczky A. Basic Principles and Clinical Applications of Neuronavigation and Intraoperative Computed Tomography, Computer Aided Surgery 1998;3(4):166-173, https://doi.org/ 14.3109/10929089809148141

15. Grunert P, Hopf N, Perneczky A. Frame-based and frameless endoscopic procedures in the third ventricle. StereotactFunctNeurosurg 68:80- 89.https:// doi.org/10.1159/000099907

16. Hellwig D, Benes L, Bertalanffy H, Bauer BL. Endoscopic stereotaxy - an eight year's experience. StereotactFunctNeurosurg. 1997;68(1-4 Pt 1):90-97. https://doi.org/10.1159/000099908

17. Barnett GH, Miller DW (1998) Brain biopsy and related procedures. In Barnett GH, Roberts DW, and Maciunas RJ (eds): Image-guided neurosurgery. St. Louis, MO: QMP Inc., pp 181-191. 